\title{
A predictive model for recurrence after upfront surgery in patients with resectable pancreatic ductal adenocarcinoma (PDAC) by using preoperative clinical data and CT characteristics
}

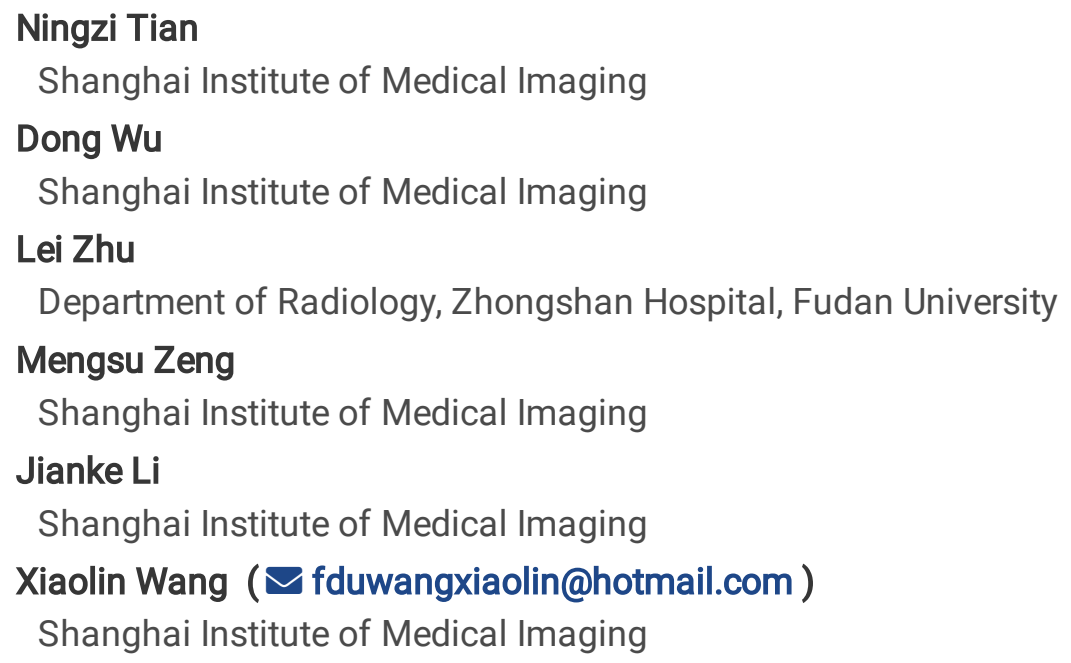

\section{Research Article}

Keywords: Pancreatic ductal adenocarcinoma, Tomography computed, Nomogram

Posted Date: February 3rd, 2022

DOI: https://doi.org/10.21203/rs.3.rs-1149955/v1

License: @ (i) This work is licensed under a Creative Commons Attribution 4.0 International License. Read Full License 


\section{Abstract \\ Background}

The purpose of this study was to develop and validate a predictive model to assess recurrence survival risk in patients with PDAC after upfront surgery by using preoperative clinical data and CT characteristics.

\section{Methods}

The predictive model was developed based on a retrospective set of 141 pancreatic cancer patients after surgery. A separate set of 77 patients was used to validate model. Between January 2017 and December 2019, all patients underwent multidetector pancreatic CT and upfront surgery. Univariable and multivariate Cox regression was used to determine the risk factors related to recurrence and then establish a nomogram to estimate the 1-year recurrence probability. Area under curve (AUC) and calibration curve are employed in evaluating the discrimination and calibration of the model.

\section{Results}

A total of 218 patients in this retrospective cohort. A recurrence model in nomogram form was developed with predictors including tumor size, tumor density in the portal vein phase, peripancreatic infiltration, suspicious metastatic lymph node, and Neutrophils/Lymphocytes ratio $\left(10^{9} / \mathrm{L}\right)$. The predictive nomogram had good discrimination capability with these predictors achieved an area under the curve for 1 year of $84.2(95 \% \mathrm{Cl}: 77.2,91.2)$ in the development set and $0.82(95 \% \mathrm{Cl}$ : $0.72,0.92)$ and $0.84(95 \% \mathrm{Cl}: 0.74,0.94)$ in the validation set for two radiologists reading respectively.

\section{Conclusions}

The model developed based on preoperative clinical data and CT characteristics of resectable pancreatic ductal adenocarcinoma patients, which can helpfully estimate the recurrence-free survival (RFS). It may be a useful tool for clinician to select optimal candidates for upfront surgery or neoadjuvant therapy.

\section{Introduction}

Pancreatic ductal adenocarcinoma (PDAC) cancer has been the fourth leading cause of cancer death worldwide, and the incidence of pancreatic cancer is increasing over the years[1,2]. The 5-year survival rate is less than $6 \%[3]$. Curative surgical resection remains considered the preferred treatment that offers long-term survival in patients with PDAC. However, patients' prognosis after curative surgical resection is dismal, which a significant proportion patient would develop locoregional and/or distant recurrence in one year[4]. Micrometastases appear to be the main cause of early recurrence, which exist before the operative resection and may contribute to the poor prognosis.

As neoadjuvant therapy potential benefits including decreasing the tumor size, sterilizing micrometastasis, increasing the rate of R0 resection and improving the probability of radical surgery, it's recommended for borderline resectable and locally advanced PDAC as initial treatment strategy to prolong survival, which has been accepted widely. As for resectable PDAC, the standard treatment option is upfront surgery, which means performing curative surgery before chemotherapy or radiation therapy, and then followed by adjuvant chemotherapy[5]. Recently, neoadjuvant therapy also has been recommended for high-risk resectable PDAC patients by National Comprehensive Cancer Network (NCCN) guidelines[6]. 
However, the criteria for patients at high-risk remains unclear. It may be an indication for these patients who will not benefit from curative surgical resection alone to accept neoadjuvant therapy.

Contrast-enhanced pancreatic CT is the primary option to assess the tumor staging and resectability before treatment[7]. Moreover, the pancreatic tumor characteristics at CT images, clinical data and laboratory parameters have potential prognostic value for patients with resectable PDAC[8]. Previous research referenced predictors focused on postsurgical factor such as tumor degree of differentiation, surgical margin, and CA19-9 decline, which couldn't be known when making treatment strategy.

Some studies had revealed that the time to recurrence from resection less than one year was significantly associated with poor overall survival[9-13]. A predictive model for evaluating risk of recurrence in one year after upfront surgery by using preoperative CT characteristics is essential for treatment decision, providing the evidence for resectable PDAC patients at high-risk choosing neoadjuvant therapy.

\section{Materials And Methods Patients' selection}

This retrospective study was approved by our hospital Ethics Committee and the need for informed patient consent was waived. We used TRIPOD (transparent reporting of a multivariable prediction model for individual prognosis or diagnosis) guidelines to guaranteeing the rigor and standard of this study [14].

This study flow chart was shown in Figure 1, descripted the patient selection process and exclusion criterion. We reviewed the contrast-enhanced pancreatic CT reports in hospital database from January 2017 to December 2019 and consecutively registered the patients with resectable PDAC. According to the NCCN criteria, the criteria of pancreatic cancer resectability is the tumor with no contact celiac artery, superior mesenteric artery, and common hepatic artery, and no contact or $\leq 180^{\circ}$ contact with the portal vein or superior mesenteric vein without vein contour irregularity. Each radiologic and medical record was reviewed by two experienced radiologists (W.D. and Z.L., with 20 and 8 years of working experience in radiology department respectively). The exclusion criteria were as follows: (a) surgery not performed or underwent palliative surgery; (b) metastases detected with other imaging scan; (c) being not PDAC by pathology confirm; (d) coexisting other malignant tumor or severe other primary diseases. Cases with incomplete clinical data also were excluded. In the final cross-sectional study sample, 218 eligible patients were enrolled. They were divided into the development set and the validation set according to the time of performing surgery.

\section{Clinical variables}

Based on previous reported, demographic and clinical variables were chosen as potential variables associated with prognosis of patients with pancreatic cancer. Preoperative laboratory variables comprised carbohydrate antigen 19-9 (CA19-9), total bilirubin, lymphocytes, neutrophils, platelets, and C-reactive protein were routinely measured within one week before surgery. About pathology findings, we recorded the following information from surgical specimens: type of pancreatic surgery, grade of differentiation, lymphvascular invasion, perineural invasion. Pathologic tumor stage was complied with the eighth edition of the American Joint Committee on Cancer (AJCC) staging system[15].

\section{CT examination and image variables}

Pancreatic cancer NCCN guidelines suggested that CT scan should contain unenhanced imaging, arterial phase (AP) and portal venous phase (PVP) imaging [6]. The Contrast-enhanced CT scan were performed by using the Aquilion ONE CT

(Toshiba Medical Systems Corporation) and Light Speed VCT (GE Healthcare). The scanning parameters were as follows: 0.5-3 mm slice thickness; in-plane resolution of $0.5 \times 0.5 \mathrm{~mm}$ or $0.625 \times 0.625 \mathrm{~mm} /$ pixel; data reconstructed at 2-5 mm 
intervals; tube voltage 100-120 kV and tube current 100-150 mA. Images were obtained after intravenous administration of $80-100 \mathrm{ml}$ of $300 \mathrm{mg}$ of iodine per milliliter of nonionic contrast material (Ultravist 300; Schering) using a power injector through an 18-gauge at a rate of $3-5 \mathrm{ml} / \mathrm{s}$. The arterial phase and portal vein phase were started at 20-35s and 6075s, respectively, after injection.

CT variables were chosen from PDAC radiology reporting template, which included tumor location, size, enhancement pattern, tumor necrosis, peripancreatic infiltration, tumor contact with major vessel, adjacent organ invasion, and suspicious metastatic lymph node[16]. The tumor density in the AP and PVP was recorded as hypo-, iso-, or hyperdense. Tumor tissue without enhance after intravenous contrast material administration was considered as necrosis area. All image variables were assessed by two radiologists (W.D. and Z.L.). They didn't know postsurgical pathologic findings and outcome data.

\section{Outcome measures}

Recurrence-free survival was defined as the date from curative surgical resection to recurrence, metastasis or death. Tumor recurrence was defined as newly detected locoregional and/or distant metastatic tumors based on the findings of $\mathrm{CT}, \mathrm{MRI}, \mathrm{PET} / \mathrm{CT}$ or US with or without increased serum carcinoembryonic antigen (CEA), carbohydrate antigen 125(CA125) or CA19-9 levels. Curative surgical resection was performed by specialized surgeon (50-arounded pancreatic cancer surgeries annually). All patients were followed up with biochemical and imaging examination assessment every 3 to 6 months until March 2021.

\section{Nomogram establishment}

Based on the clinical and imaging predictor, the final predictive model was established. All covariates in Cox model were selected as risk factor by stepwise regression. The nomogram was generated with the independent risk factors for predicting the 1-year RFS probability. Time-dependent receiver operating characteristic curve was plotted to assess the discrimination of our model in development and validation sets respectively. Meanwhile, we evaluated the agreement between predicted and observed probabilities.

\section{Statistical analysis}

All statistical analyses were performed with SPSS software (version 20.0) and RStudio (version 4.0.4). Patient characteristics of this study sample were described by means and standard deviations for continuous variables and frequencies and percentages for categorical variables. The consistency evaluation between the two sets was applied with Fisher exact, $t$ test, $\chi^{2}$ validation, validation, or analysis of variance according to data type. Multivariable Cox proportional hazards models to filter risk factors association with RFS. The hazard ratio (HR) and corresponding $95 \%$ confidence intervals were estimated. $P<0.05$ was considered to indicate statistical significance.

In the development set, the model was built for predicting 1-year RFS probability with stepwise elimination. We used calibration capability plot to assess the model performing. Probabilities of 1 -year recurrence were read according to the nomography.

\section{Results}

\section{Patient characteristics}

All 456 patients with PDAC were screened and 270 patients satisfied with eligibility. 52 patients were excluded for coexisting other malignant tumor, severe primary diseases, and clinical data incomplete. Finally, the study sample comprised 218 patients and was divided into the development set $(n=171$; mean age, 63.43years $\pm 9.25[$ standard 
deviation]; 87 male) and the validation set ( $=77$; mean age, 64.49years $\pm 10.40 ; 41$ male). The patient characteristics are summarized as following (Table 1). 
Table 1

Patients' characteristics of the study sample.

\begin{tabular}{|c|c|c|c|}
\hline Characteristic & Development Set(n=141) & Validation Set(n=77) & $P$ Value \\
\hline Age (years) $^{a}$ & $63.43 \pm 9.25$ & $64.49 \pm 10.40$ & $0.277^{\#}$ \\
\hline Gender ${ }^{\square}$ & $87(61.70)$ & $41(53.25)$ & 0.226 \\
\hline Male & $54(38.30)$ & $36(46.75)$ & \\
\hline \multicolumn{4}{|l|}{ Female } \\
\hline Tumor location & & & 0.200 \\
\hline Head & $88(62.41)$ & $40(51.95)$ & \\
\hline Body & $19(13.48)$ & $17(22.08)$ & \\
\hline Tail & $34(24.11)$ & $20(25.97)$ & \\
\hline \multicolumn{4}{|l|}{ Laboratory results } \\
\hline Cancer antigen 19-9(U/ml) & 141.2(0.6-7767) & $92.1(2-10000)$ & 0.573 \\
\hline Bilirubin $(\mu \mathrm{mol} / \mathrm{L})$ & 13.8(3.7-436.1) & $13.2(3.8-450.4)$ & 0.334 \\
\hline Neutrophils/Lymphocytes $\left(10^{9} / \mathrm{L}\right)$ & $2.49(0.52-13.25)$ & $2.41(0.91-4.99)$ & 0.479 \\
\hline Platelets/Lymphocytes (109/L) & $134.12(61.07-463.33)$ & $137.06(2.4-428)$ & 0.985 \\
\hline C-reactive protein(mg/L) & $1.2(0.3-32.9)$ & $1.6(0.3-381.2)$ & 0.294 \\
\hline Adjuvant therapy performed ${ }^{\star}$ & 106(75.18) & $59(76.62)$ & 0.812 \\
\hline Type of pancreatic surgery ${ }^{£}$ & & & 0.924 \\
\hline Standard pancreaticoduodenectomy & $54(38.30)$ & $29(37.66)$ & \\
\hline Standard distal pancreatectomy & $52(36.88)$ & $28(36.36)$ & \\
\hline Extended pancreaticoduodenectomy & $26(18.44)$ & $14(18.18)$ & \\
\hline Extended distal pancreatectomy & $8(5.67)$ & $6(7.79)$ & \\
\hline Standard total pancreatectomy & $1(0.71)$ & $0(0.00)$ & \\
\hline Negative resection margin (R0) & $140(99.29)$ & 760 & 0.663 \\
\hline Primary tumor $(T)$ stage & & & 0.900 \\
\hline
\end{tabular}

Data are medians, with ranges in parentheses, unless otherwise specified.

a Data are means \pm standard deviations

${ }^{*}$ Data are numbers, with percentages in parentheses.

${ }^{£}$ Type of surgery was classified as standard surgery or extended surgery involving concomitant vein or additional organ resection.

\#Fisher's exact test, $\chi^{2}$ test or one-way analysis of variance were used to compared data, excepted where indicated. 


\begin{tabular}{|c|c|c|c|}
\hline Characteristic & Development Set(n=141) & Validation Set(n=77) & $P$ Value \\
\hline T1 & $44(31.21)$ & $22(28.57)$ & \\
\hline T2 & $79(56.03)$ & $44(57.14)$ & \\
\hline T3 & 18(12.76) & 11(14.29) & \\
\hline Regional lymph node $(\mathrm{N})$ stage & & & 0.064 \\
\hline NO & $80(56.74)$ & $54(70.13)$ & \\
\hline $\mathrm{N} 1$ & $53(37.59)$ & 17(22.08) & \\
\hline N2 & $8(5.67)$ & $6(7.79)$ & \\
\hline AJCC prognostic stage group & & & 0.090 \\
\hline IA & $29(20.57)$ & $12(15.58)$ & \\
\hline IB & $41(29.08)$ & $32(41.56)$ & \\
\hline$\| A$ & $10(7.09)$ & $8(10.39)$ & \\
\hline IIB & $51(36.17)$ & 17(22.08) & \\
\hline III & $9(6.38)$ & $5(6.49)$ & \\
\hline IV & $1(0.71)$ & $3(3.90)$ & \\
\hline Tumor differentiation & & & 0.108 \\
\hline Well differentiated & $0(0.00)$ & $0(0.00)$ & \\
\hline Moderately differentiated & $70(49.65)$ & $47(61.04)$ & \\
\hline Poorly or undifferentiated & $71(50.35)$ & $30(38.96)$ & \\
\hline Lymphovascular or microvascular invasion present & 27(19.15) & 18(23.38) & 0.461 \\
\hline \multicolumn{4}{|c|}{ Data are medians, with ranges in parentheses, unless otherwise specified. } \\
\hline \multicolumn{4}{|l|}{ a Data are means \pm standard deviations } \\
\hline \multicolumn{4}{|l|}{${ }^{*}$ Data are numbers, with percentages in parentheses. } \\
\hline \multicolumn{4}{|c|}{$\begin{array}{l}\text { EType of surgery was classified as standard surgery or extended surgery involving concomitant vein or additional } \\
\text { organ resection. }\end{array}$} \\
\hline
\end{tabular}

As shown in Tables 1, the distribution of laboratory results is similar in the two sets. Because of cross-sectional study, it's can be explained that follow-up duration in the development set was longer than the validation set (median, 33months [rang, 2-48 months] vs median, 18months[rang, 6-24 months]; $P<0.001)$. Tumors recurred in 96 of 141 (68.1\%) patients in the development set and 44 of 77 (57.1\%) patients in the validation set during follow-up period. Median RFS was significantly different between the development and validation sets (median, 17.84 months [rang, 1.08-48.03 months] vs median, 14.23 months [rang, 2.37-24.48 months]; $P=0.007$ ). Adjuvant therapy performed was same frequent in the development and validation sets ( $75.18 \%$ [ 106 of 141 ] vs $76.62 \%$ [ 59 of 77$]$; $P=0.812$ ).

\section{Univariate and multiple Cox regression analysis}


Through Cox proportional hazard analysis, independent risk factors were selected associated with recurrence including: tumor size (hazard ratio [HR], 1.417; $95 \% \mathrm{Cl}$ : 1.098, 1.495; $P=0.002)$, tumor density in the portal vein phase (HR, -0.940 ; 95\% Cl: 0.424, 0.844; $P=0.003)$, peripancreatic infiltration (HR, 4.151; 95\% Cl: 2.077, 8.298; $P=0.000)$, suspicious

metastatic lymph node (HR, 2.561; $95 \% \mathrm{Cl}$ : 1.653, 3.967; $P=0.000)$, Neutrophils/Lymphocytes ratio $\left(10^{9} / \mathrm{L}\right)(\mathrm{HR}, 1.111 ; 95 \%$ Cl: 1.016, 1.215; $P=0.020$ ) (Table 2). 
Table 2

Using Cox proportional hazard analyses for postsurgical RFS in development set.

\begin{tabular}{|c|c|c|c|c|c|c|}
\hline \multirow[t]{2}{*}{ Parameter } & \multicolumn{3}{|c|}{$\begin{array}{l}\text { Univariable Cox Proportional Hazard } \\
\text { Analysis }\end{array}$} & \multicolumn{3}{|c|}{$\begin{array}{l}\text { Multivariable Cox Proportional Hazard } \\
\text { Analysis }\end{array}$} \\
\hline & $\begin{array}{l}\text { Regression } \\
\text { Coefficient }\end{array}$ & Hazard Ratio & $\begin{array}{l}P \\
\text { Value }\end{array}$ & $\begin{array}{l}\text { Regression } \\
\text { Coefficient }\end{array}$ & Hazard Ratio & $\begin{array}{l}P \\
\text { Value }\end{array}$ \\
\hline Age & -0.016 & $0.984(0.963,1.006)$ & 0.157 & & & \\
\hline Male sex & 0.063 & $1.065(0.705,1.608)$ & 0.766 & & & \\
\hline Tumor size(cm) & 0.349 & $1.417(1.222,1.644)$ & 0.000 & -0.248 & $1.277(1.098,1.495)$ & 0.002 \\
\hline Dominant location & & & 0.165 & & & \\
\hline Head & -0.105 & $0.901(0.566,1.434)$ & 0.659 & & & \\
\hline Body & -0.721 & $0.486(0.227,1.042)$ & 0.486 & & & \\
\hline Tail & 1 & 1[reference] & $\ldots$ & & & \\
\hline Tumor density in AP & & & 0.747 & & & \\
\hline hyperdense & 1 & 1 [reference] & $\ldots$ & & & \\
\hline Isodense & 0.301 & $1.351(0.407,4.491)$ & 0.623 & & & \\
\hline Hypodense & 0.20 & $1.020(0.373,2.787)$ & 0.969 & & & \\
\hline Tumor density in PVP & & & 0.000 & -0.940 & $0.598(0.424,0.844)$ & 0.003 \\
\hline hyperdense & 1 & 1 [reference] & $\ldots$ & & & \\
\hline Isodense & 0.407 & $1.503(0.714,3.163)$ & 0.283 & & & \\
\hline Hypodense & 1.141 & $3.129(1.526,6.416)$ & 0.002 & & & \\
\hline Tumor necrosis (Yes/No) & 0.104 & $1.110(0.451,2.733)$ & 0.821 & & & \\
\hline $\begin{array}{l}\text { Peripancreatic infiltration } \\
\text { (Yes/No) }\end{array}$ & -1.489 & $0.226(0.116,0.438)$ & 0.000 & 1.423 & $4.151(2.077,8.298)$ & 0.000 \\
\hline $\begin{array}{l}\text { Contact to SMV or PV } \\
\text { Adjacent Organ invasion }\end{array}$ & -0.228 & $0.431(0.451,1.405)$ & 0.431 & & & \\
\hline $\begin{array}{l}\text { Suspicious metastatic } \\
\text { lymph node }\end{array}$ & -1.089 & $0.336(0.223,0.507)$ & 0.000 & 0.940 & $2.561(1.653,3.967)$ & 0.000 \\
\hline Cancer antigen 19-9 & 0.000 & $1(1,1)$ & 0.009 & & & \\
\hline Bilirubin & 0.003 & $1.003(1.001,1.005)$ & 0.011 & & & \\
\hline $\begin{array}{l}\text { Neutrophils/Lymphocytes } \\
\left(10^{9} / \mathrm{L}\right)\end{array}$ & 0.138 & $1.148(1.066,1.237)$ & 0.000 & 0.105 & $1.111(1.016,1.215)$ & 0.020 \\
\hline $\begin{array}{l}\text { Platelets/Lymphocytes } \\
\left(10^{9} / \mathrm{L}\right)\end{array}$ & 0.000 & $1(1,1)$ & 0.835 & & & \\
\hline C-reactive protein(mg/L) & -0.042 & $0.959(0.905,1.016)$ & 0.159 & & & \\
\hline
\end{tabular}




\section{Nomogram}

The nomogram was established based on tumor size, tumor density in PVP, suspicious metastatic lymph nodes, peripancreatic tumor infiltration and NLR (Figure 2). In the development set, discrimination capability of model with the AUC of $0.84(95 \% \mathrm{Cl}: 0.77,0.91)$ is good and the calibration slope is 0.99 . The probability of 1 -year recurrence can be read from the nomogram directly. For example, a woman is with resectable PDAC. A diameter $1.4 \mathrm{~cm}$ mass (5 points) in pancreatic head appearing hyperdense (0 points) in PVP. There is no suspicious metastatic lymph node (0 points), no peripancreatic tumor infiltration (0 points) and NLR of 1.12 (5 points). The total nomogram points are 10 and a very low $(<0.1)$ probability of 1-year recurrence (Figure 3). For another case, a resectable PDAC patients with a $4.6 \mathrm{~cm}(35$ points)mass, hypodense in PVP (38 points), suspicious metastatic lymph node (38.5 points), peripancreatic tumor infiltration (58 points) and NLR of 2.96 (13 points) would have a total points of 182.5 and a 0.8 probability of 1-year recurrence (Figure 4).

\section{Nomogram performance assessment}

Our nomogram showed good discrimination capability with the AUC for predicting 1-year RFS probability of $0.82(95 \% \mathrm{Cl}$ : $0.72,0.92)$ for one reader and $0.84(95 \% \mathrm{Cl}: 0.74,0.94)$ for another reader in validation set. Calibration curves showed the agreement between predicted and observed probabilities of 1-year RFS after upfront surgery in both sets (Figure 5).

\section{Discussion}

Our study established a predictive model to estimating recurrence survival in patients with Pancreatic ductal adenocarcinoma, based on the routinely measured clinical factors and tumor features at CT images available within two weeks before the surgery. Previous predictive models had limited clinical utility as depending on postsurgical pathology findings to some extent $[13,17,18]$. Having a simple preoperative prognostic model could potentially ensure a better selection of optimal candidates for upfront surgery.

The performance of this model was satisfactory in discrimination and calibration aspects in both the development and validation sets. Factors in the model were tumor size (hazard ratio [HR]1.277; $P=0.002$ ), tumor density in the portal venous phase (hazard ratio $[\mathrm{HR}] 1.277 ; P=0.002$ ), suspicious metastatic lymph node(hazard ratio $[\mathrm{HR}] 2.561 ; P<0.001$ ), peripancreatic infiltration(hazard ratio $[H R] 4.151 ; P<0.001)$ and NLR (hazard ratio $[H R] 1.111 ; P=0.020$ ). These imaging and clinical features indicated the tumor development and progression of PDAC. The risk nomogram points reliably predicted discrimination capability of 0.84 calculated from the areas under the receiver operating characteristic curve in the validation set. The five factors we used to predict 1-year recurrence risk are easily acquired in clinical datasets. So, this nomogram can be provided as an accessible tool for clinicians to assess patients' risk of recurrence. When patients' risk of recurrence in one year assessed is low, the clinician may suggest upfront surgery. For patients with high risk, neoadjuvant therapy may be needed [19].

Preoperative NLR as an only clinical factor is in our model. The previous studies have provided evidence that inflammation is participated in outcome in patients with cancer. The neutrophils play important roles in systemic inflammatory response, which promote tumor growth, facilitate tumorigenesis, metastasis and stimulate tumor angiogenesis[20,21]. According to prior studies, NLR is a predictive marker in survival prognosis of pancreatic invasive carcinoma[21-23]. This relationship could explain the correlation between high NLR and short RFS in current study.

CA19-9, CEA and CA125 are commonly considered as tumor biomarkers for the prognosis of pancreatic cancer, among which CA19-9 is the most valuable factor used for auxiliary diagnosis and recurrence monitoring and correlated with clinical course of disease [24-26]. However, in our study, the CA19-9 is excluded from the predictive model. One reason may be that CA19-9 level can also elevated in some patients with biliary infection, inflammation and obstruction, which confound the survival outcome. 
As non-invasive imaging assessment, now contrast-enhanced pancreatic CT scans play an important part when decide treatment regimen for patients with pancreatic cancer. Our study shows that CT characteristics such as tumor size, suspicious metastatic lymph nodes, peripancreatic tumor infiltration, and tumor density in PVP can helping us predicting adverse outcomes, which are independent prognosis factors of RFS.

Several limitations in this study should be acknowledged. First, retrospective single-institution study was more prone to bias than prospective study, despite our efforts to minimize selection bias and avoid bias from missing data. Second, during the follow-up period, the consistency in determining recurrence in each patient is absent. Only a few patients had recurrence masses that were confirmed by pathologic finding. In many other cases, it's according to symptoms, the increasing of tumor biomarkers or radiologic findings to diagnose disease relapse. In addition, a longer follow-up period is needed and enrolled patients rechecked in scheduled visits should be ensured. Third, in our predictive model, a critical point for distinguish between low-risk and high-risk groups need to furtherly determined.

\section{Conclusions}

The model developed mainly based on preoperative clinical data and CT characteristics of pancreatic ductal adenocarcinoma patients can helpfully estimate the RFS, which may be a useful tool for clinician to select patients for upfront surgery or neoadjuvant therapy.

\section{Abbreviations}

PDAC

Pancreatic ductal adenocarcinoma

CT

Computed tomography

RFS

Recurrence-free survival

MRI

Magnetic resonance imaging

PET

positron emission tomography

AFP

Alpha-fetoprotein

CA19-9

Carbohydrate antigen 19-9

CEA

Carcinoembryonic antigen

CA125

Carbohydrate antigen 125

NLR

Neutrophils/Lymphocytes ratio

AP

arterial phase

PVP

portal venous phase.

\section{Declarations}




\section{Ethics approval and consent to participate}

Our study was approved by the Ethics Committee of the FUZS hospital and requirement for informed patient consent was waived as a retrospective study. This study was not referred patient interactions.

\section{Consent for publication}

Not applicable.

\section{Availability of data and materials}

Due to patient privacy protection, materials and data are not publicly available but are available from the corresponding author for reasonable request.

\section{Competing interests}

The authors declare that they have no competing interests.

\section{Funding}

None

\section{Authors' contributions}

All authors read and approved the final manuscript. NZT, DW and LZ collected clinical and imaging data, MSZ, JKL and XLW participated in the design of this study. All authors read the final manuscript and approval for publication.

\section{Acknowledgements}

None

\section{Authors' information}

Not applicable.

\section{Author details}

${ }^{1}$ Shanghai Institute of Medical Imaging

2 Department of Radiology, Zhongshan Hospital, Fudan University

No. 180 Fenglin Road, Xuhui District, Shanghai 200032, China, Postcode: 200032

\section{References}

1. Lin QJ, Yang F, Jin C, Fu DL. Current status and progress of pancreatic cancer in China. World J Gastroenterol. 2015; 21(26):7988-8003.

2. He J, Ahuja N, Makary MA, Cameron JL, Eckhauser FE, Choti MA, Hruban RH, Pawlik TM, Wolfgang CL. 2564 resected periampullary adenocarcinomas at a single institution: trends over three decades. HPB (Oxford). 2014; 16(1):83-90.

3. Siegel RL, Miller KD, Fuchs HE, Jemal A. Cancer Statistics, 2021. CA Cancer J Clin. 2021; 71(1):7-33.

4. Groot VP, Rezaee N, Wu W, Cameron JL, Fishman EK, Hruban RH, Weiss MJ, Zheng L, Wolfgang CL, He J. Patterns, Timing, and Predictors of Recurrence Following Pancreatectomy for Pancreatic Ductal Adenocarcinoma. Ann Surg. 
2018; 267(5):936-945.

5. Tempero MA, Malafa MP, Chiorean EG, Czito B, Scaife C, Narang AK, Fountzilas C, Wolpin BM, Al-Hawary M, Asbun H, et al. Pancreatic Adenocarcinoma, Version 1.2019. J Natl Compr Canc Netw. 2019; 17(3):202-210.

6. Tempero MA, Malafa MP, Al-Hawary M, Behrman SW, Benson AB, Cardin DB, Chiorean EG, Chung V, Czito B, Del CM, et al. Pancreatic Adenocarcinoma, Version 2.2021, NCCN Clinical Practice Guidelines in Oncology. J Natl Compr Canc Netw. 2021; 19(4):439-457.

7. Mizrahi JD, Surana R, Valle JW, Shroff RT. Pancreatic cancer. Lancet. 2020; 395(10242):2008-2020.

8. Koay EJ, Lee Y, Cristini V, Lowengrub JS, Kang Y, Lucas F, Hobbs BP, Ye R, Elganainy D, Almahariq M, et al. A Visually Apparent and Quantifiable CT Imaging Feature Identifies Biophysical Subtypes of Pancreatic Ductal Adenocarcinoma. Clin Cancer Res. 2018; 24(23):5883-5894.

9. Furukawa K, Shiba H, Hamura R, Haruki K, Fujiwara Y, Usuba T, Nakabayashi Y, Misawa T, Okamoto T, Yanaga K. Prognostic Factors in Patients With Recurrent Pancreatic Cancer: A Multicenter Database Analysis. Anticancer Res. 2020; 40(1):293-298.

10. Kim YI, Song KB, Lee YJ, Park KM, Hwang DW, Lee JH, Shin SH, Kwon JW, Ro JS, Kim SC. Management of isolated recurrence after surgery for pancreatic adenocarcinoma. Br J Surg. 2019; 106(7):898-909.

11. Groot VP, Gemenetzis G, Blair AB, Rivero-Soto RJ, Yu J, Javed AA, Burkhart RA, Rinkes I, Molenaar IQ, Cameron JL, et al. Defining and Predicting Early Recurrence in 957 Patients With Resected Pancreatic Ductal Adenocarcinoma. Ann Surg. 2019; 269(6):1154-1162.

12. Narayanan S, Almasri S, Zenati M, Nassour I, Chopra A, Rieser C, Smith K, Oyefusi V, Daum T, Bahary N, et al. Predictors of early recurrence following neoadjuvant chemotherapy and surgical resection for localized pancreatic adenocarcinoma. J Surg Oncol. 2021.

13. Kim NH, Kim HJ. Preoperative risk factors for early recurrence in patients with resectable pancreatic ductal adenocarcinoma after curative intent surgical resection. Hepatobiliary Pancreat Dis Int. 2018; 17(5):450-455.

14. Moons KG, Altman DG, Reitsma JB, loannidis JP, Macaskill P, Steyerberg EW, Vickers AJ, Ransohoff DF, Collins GS. Transparent Reporting of a multivariable prediction model for Individual Prognosis or Diagnosis (TRIPOD): explanation and elaboration. Ann Intern Med. 2015; 162(1):W1-W73.

15. Amin MB, Greene FL, Edge SB, Compton CC, Gershenwald JE, Brookland RK, Meyer L, Gress DM, Byrd DR, Winchester DP. The Eighth Edition AJCC Cancer Staging Manual: Continuing to build a bridge from a population-based to a more "personalized" approach to cancer staging. CA Cancer J Clin. 2017; 67(2):93-99.

16. Al-Hawary MM, Francis IR, Chari ST, Fishman EK, Hough DM, Lu DS, Macari M, Megibow AJ, Miller FH, Mortele KJ, et al. Pancreatic ductal adenocarcinoma radiology reporting template: consensus statement of the Society of Abdominal Radiology and the American Pancreatic Association. Radiology. 2014; 270(1):248-260.

17. Adamu M, Nitschke P, Petrov P, Rentsch A, Distler M, Reissfelder C, Welsch T, Saeger HD, Weitz J, Rahbari NN. Validation of prognostic risk scores for patients undergoing resection for pancreatic cancer. Pancreatology. 2018; 18(5):585-591.

18. Strijker M, Chen JW, Mungroop TH, Jamieson NB, van Eijck CH, Steyerberg EW, Wilmink JW, Groot KB, van Laarhoven HW, Besselink MG. Systematic review of clinical prediction models for survival after surgery for resectable pancreatic cancer. Br J Surg. 2019; 106(4):342-354.

19. Versteijne E, Suker M, Groothuis K, Akkermans-Vogelaar JM, Besselink MG, Bonsing BA, Buijsen J, Busch OR, Creemers GM, van Dam RM, et al. Preoperative Chemoradiotherapy Versus Immediate Surgery for Resectable and Borderline Resectable Pancreatic Cancer: Results of the Dutch Randomized Phase III PREOPANC Trial. J Clin Oncol. 2020; 38(16):1763-1773. 
20. Zhang X, Zhang W, Yuan X, Fu M, Qian H, Xu W. Neutrophils in cancer development and progression: Roles, mechanisms, and implications (Review). Int J Oncol. 2016; 49(3):857-867.

21. Guthrie GJ, Charles KA, Roxburgh CS, Horgan PG, Mcmillan DC, Clarke SJ. The systemic inflammation-based neutrophil-lymphocyte ratio: experience in patients with cancer. Crit Rev Oncol Hematol. 2013; 88(1):218-230.

22. Gemenetzis G, Bagante F, Griffin JF, Rezaee N, Javed AA, Manos LL, Lennon AM, Wood LD, Hruban RH, Zheng L, et al. Neutrophil-to-lymphocyte Ratio is a Predictive Marker for Invasive Malignancy in Intraductal Papillary Mucinous Neoplasms of the Pancreas. Ann Surg. 2017; 266(2):339-345.

23. Matsumoto T, Okabayashi T, Sui K, Morita S, Iwata J, Shimada Y. Preoperative neutrophili-to-lymphocyte ratio is useful for stratifying the prognosis of tumor markers-negative pancreatic cancer patients. Am J Surg. 2020; 219(1):93-98.

24. Sakamoto T, Saito H, Uchinaka El, Morimoto M, Amisaki M, Tokuyasu N, Honjo S, Ashida K, Fujiwara Y. The Combination of Neutrophil-to-lymphocyte Ratio and Serum Carbohydrate Antigen 19-9 Level as a Prognostic Indicator in Patients with Recurrent Pancreatic Cancer. Anticancer Res. 2018; 38(9):5497-5503.

25. Hong S, Song KB, Hwang DW, Lee JH, Lee W, Kwon J, Park Y, Kim SC. Preoperative serum carbohydrate antigen 19-9 levels predict early recurrence after the resection of early-stage pancreatic ductal adenocarcinoma. Annals of HepatoBiliary-Pancreatic Surgery. 2021; 25(1):S77.

26. Kurahara H, Maemura K, Mataki Y, Sakoda M, lino S, Kawasaki Y, Arigami T, Mori S, Kijima Y, Ueno S, et al. A Therapeutic Strategy for Resectable Pancreatic Cancer Based on Risk Factors of Early Recurrence. Pancreas. 2018; 47(6):753-758.

\section{Figures}




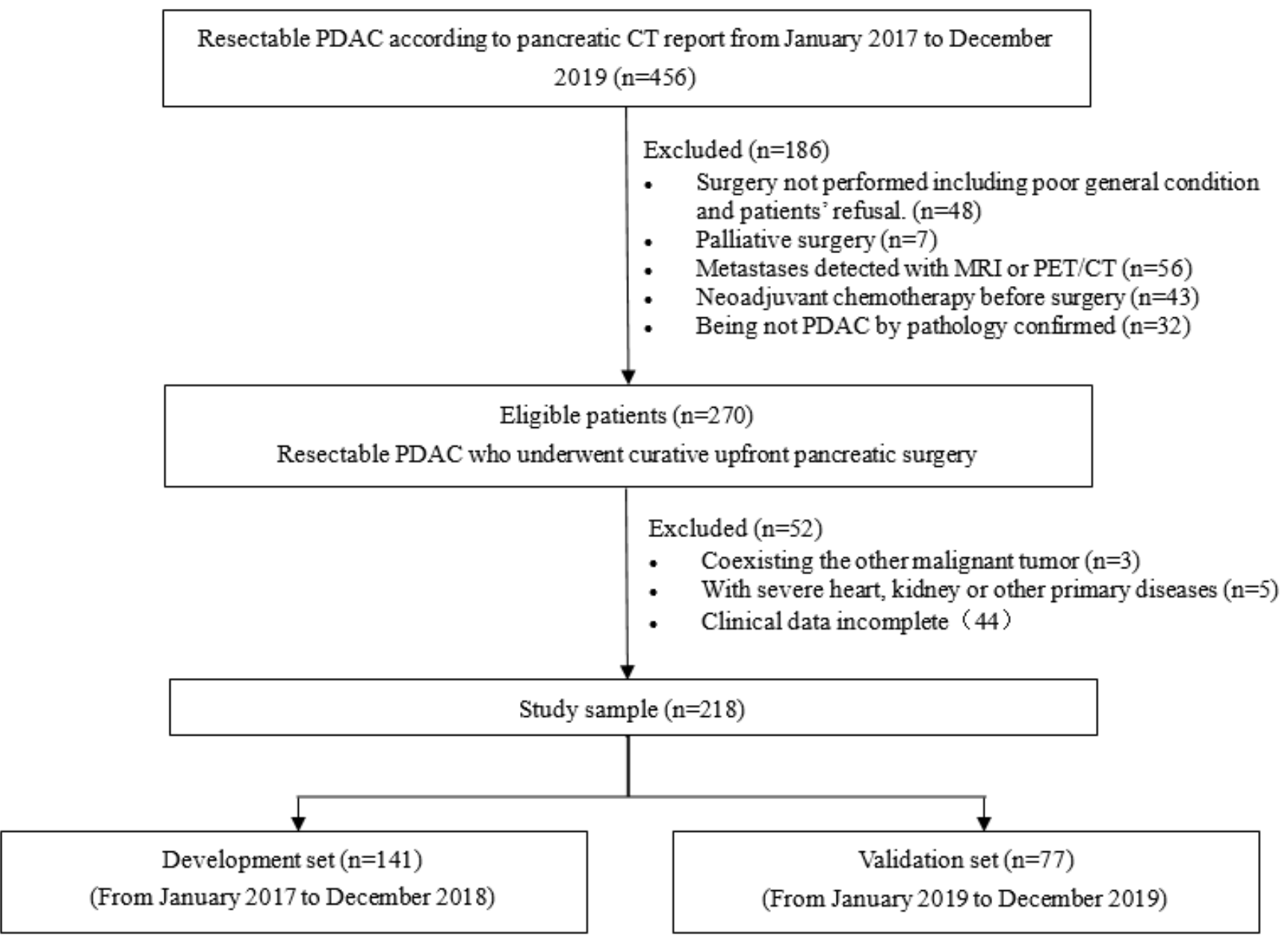

Figure 1

Study flow chart for development and validation set. After exclusion, 218 of 454 patients were identified in our study.

Points

Size

Density in PVP

Suspicious metastatic lymph nodes

Peripancreatic tumor infiltration

NLR

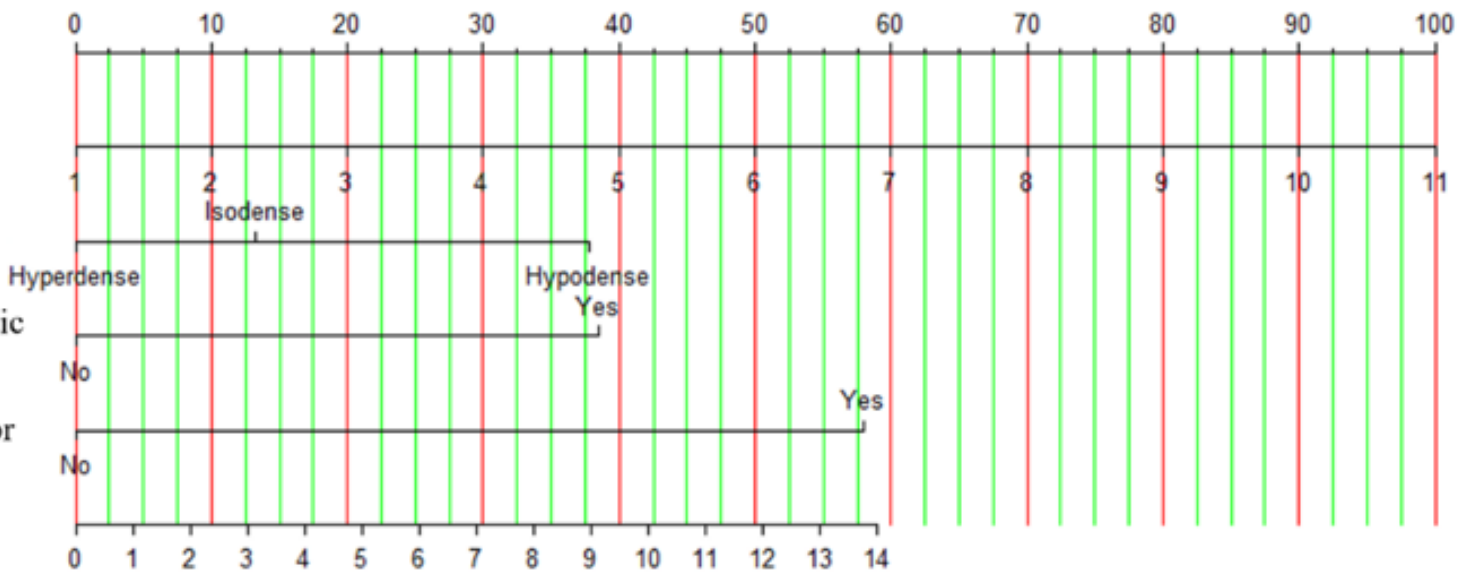

Total points

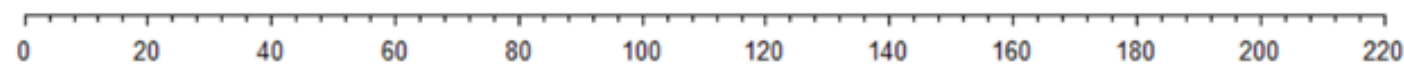

1-year RFS probability

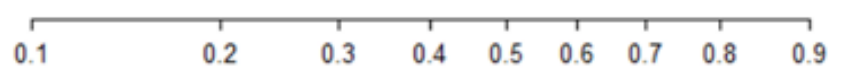

Figure 2 

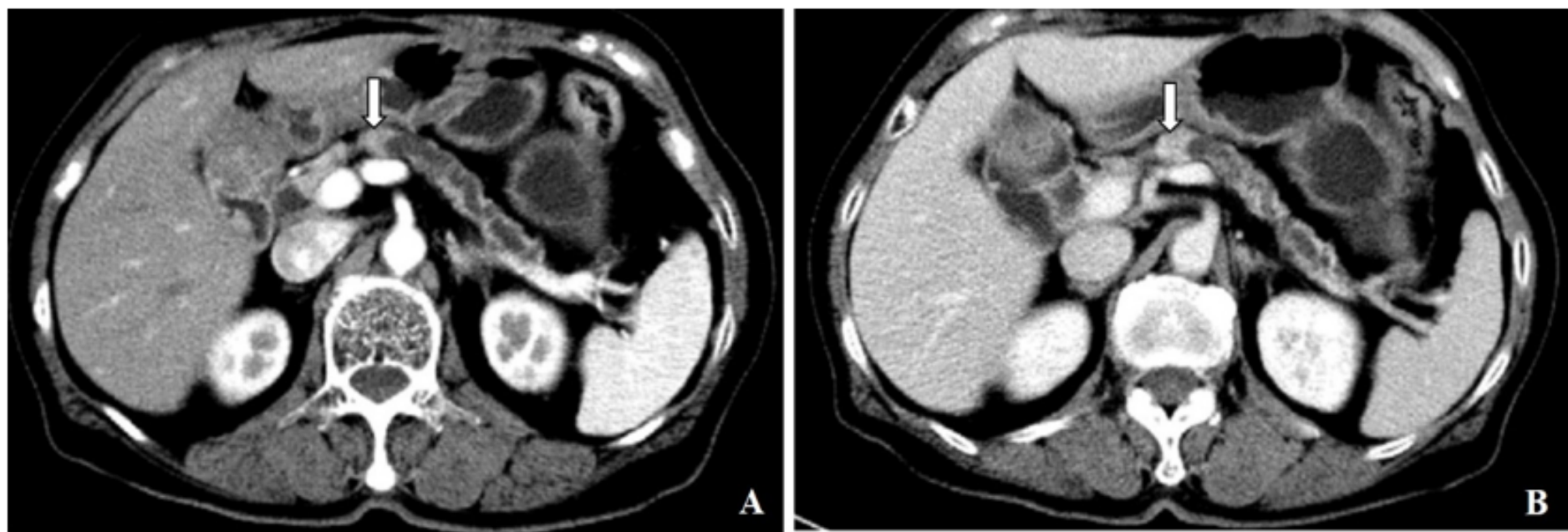

\section{Figure 3}

A 73-year-old woman with no discomfort and was admitted to our hospital for pancreatic mass detected by CT examination. Contrast-enhanced CT images show a mass in pancreatic head (arrow) with diameter 1.4-cm, hyperdense in (A) arterial phase and (B) portal venous phase. Tumor infiltration and enlarged lymph nodes are absent. NLR is 1.12. patient was alive for $\mathbf{4 0}$ months until our last follow-up after standard pancreaticoduodenectomy with no tumor recurrence.

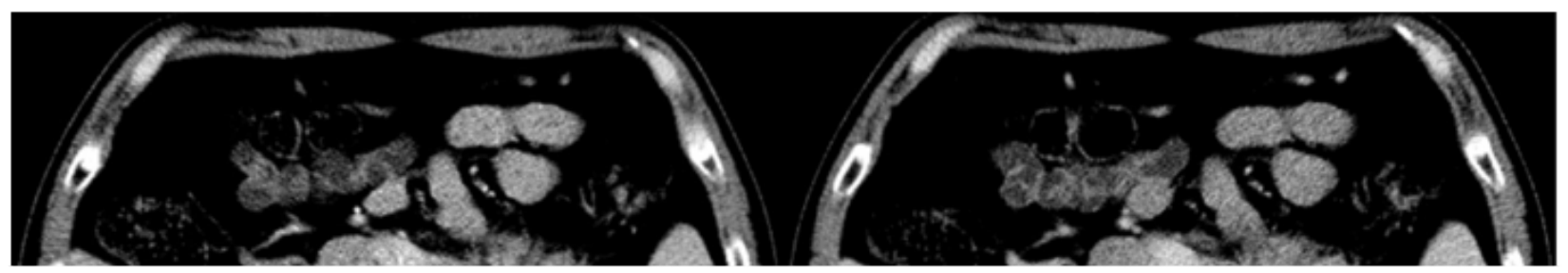




\section{Figure 4}

A 62-year-old man with elevated CA19-9. Preoperative CT detected a solid lesion in pancreatic tail. Moderately differentiated pancreatic ductal adenocarcinoma was confirmed after extended distal pancreatectomy. Contrastenhanced CT images demonstrate a 4.6-cm hypodense mass in pancreatic tail (arrow) (C凶in (A) arterial phase and (B) portal venous phase. Peripancreatic infiltration is appeared(arrowhead). Two enhanced suspicious metastatic lymph nodes (D) are observed (arrow). NLR is 2.96. Tumor recurrence occurrence 5.6 months and died 15.1 months after surgery.
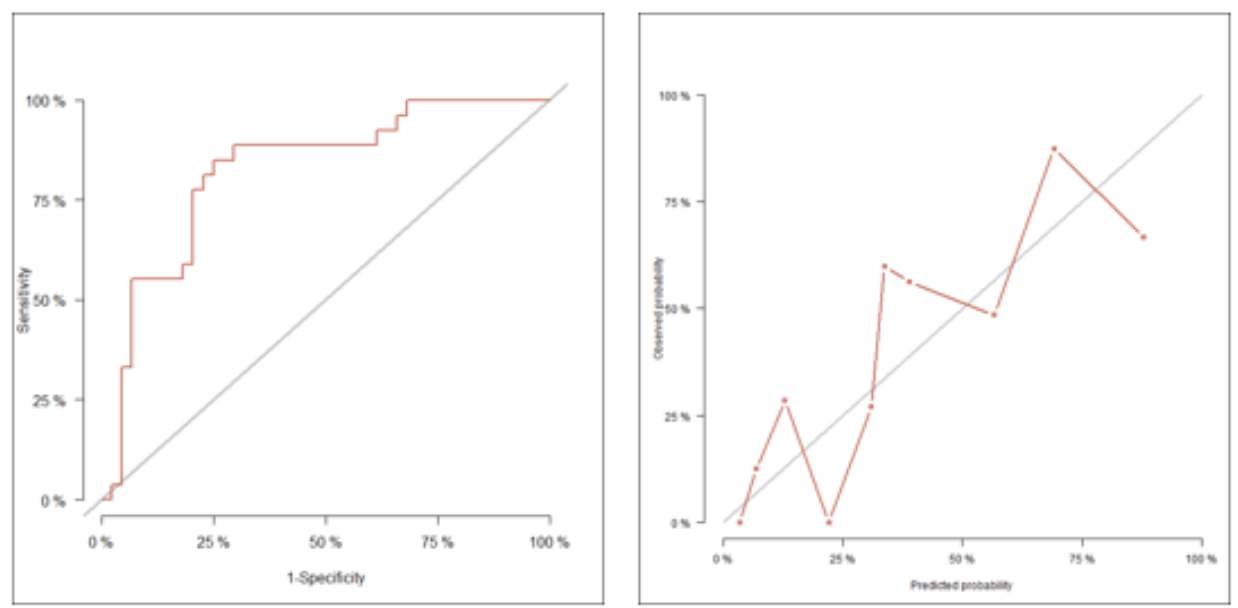

A
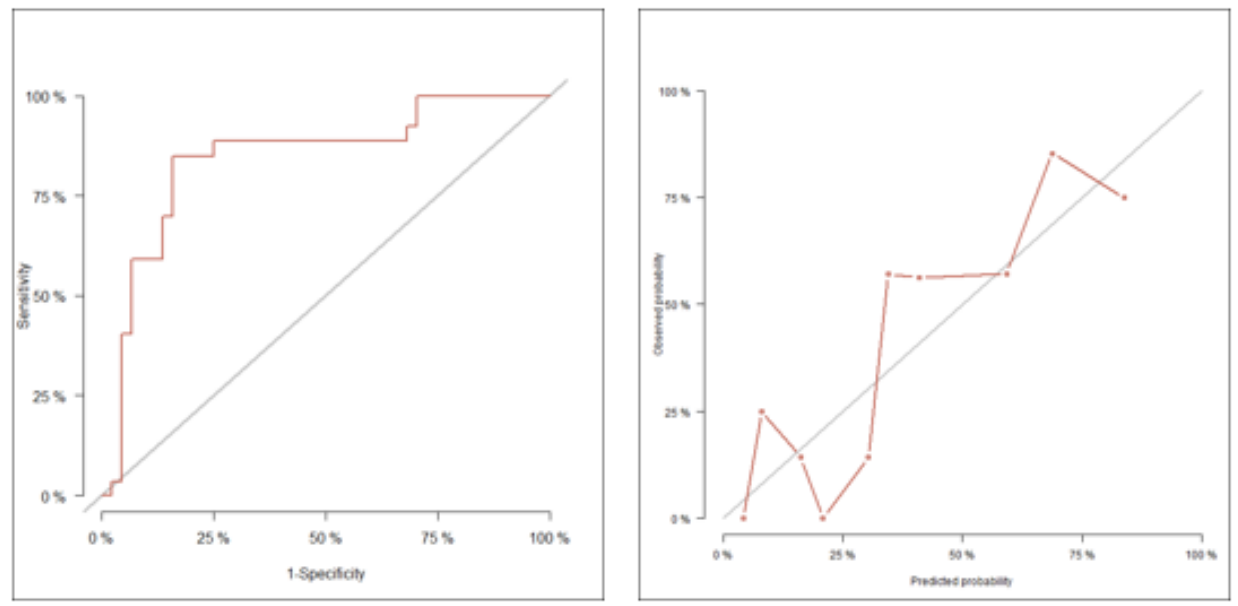

B

\section{Figure 5}

AUC and Calibration plots compare probability of 1-year RFS between predicted and observed in validation set interpretations of reader1(A)and reader2(B). 